\section{In-situ, non-destructive acoustic characterization of solid state electrolyte cells}

Robert D. Schmidt ${ }^{a}$ and Jeffrey Sakamoto ${ }^{\mathrm{a}, *}$

a Department of Mechanical Engineering, University of Michigan, Ann Arbor, MI

\section{5, USA}

* Corresponding author. E-mail address: jeffsaka@umich.edu (J. Sakamoto); Telephone: (734) 763-2219; FAX: (734) 647-9379 


\section{Keywords}

acoustic; elastic moduli; microcracks; dendrite

\section{Introduction}

An approach to improve the performance and safety of batteries is replace stateof-the-art liquid electrolytes with solid-state ceramic electrolytes [1-3]. Myriad solid-state electrolytes are currently under development, including polymers [4] and ceramics such as sulfides, oxides, and phosphates [1,2].

Garnet of nominal formulation $\mathrm{Al}_{0.25} \mathrm{Li}_{(6.25)} \mathrm{La}_{3} \mathrm{Zr}_{2} \mathrm{O}_{12}$ (LLZO), is a promising candidate electrolyte and is believed to be stable against metallic Li and has adequate room temperature ionic conductivity to replace liquid electrolytes $[1,2,5]$. While these attributes are important in the context of technology maturation, the maximum current tolerable ionic current density (or critical current density - CCD) must approach $\sim 1 \mathrm{~mA}$ $\mathrm{cm}^{-2}$ or values comparable to liquid-based electrolytes.

Recent studies [6-8] indicated that the CCD of LLZO may be $<1 \mathrm{~mA} \mathrm{~cm}^{-2}$ at room temperature when employing symmetric Li anode cells. It is believed that Li metal dendrites initiate and propagate between cell electrodes, thus causing short-circuiting. Little is known about the Li metal initiation and propagation in LLZO as a function of current density. The most relevant work was conducted decades ago where the initiation and propagation of $\mathrm{Na}$ metal through sodium beta-alumina (SBA) solid electrolyte was studied [9]. The majority of the investigations involved DC cycling of molten $\mathrm{Na}$ at elevated temperature $\left(300^{\circ} \mathrm{C}\right)[10-12]$. Several models were established to describe the phenomenon; some were based on experimental data indicating the mechanical properties, such as the fracture toughness and elastic modulus, had a 
profound effect on governing the maximum tolerable current density [10-12]. Above the critical current density, Na metal propagated as dendrites or filaments through the SBA microstructure [12]. Thus, based on this previous work, we believe that a characterization approach that measures changes in mechanical properties resulting from the introduction of microstructural defects could elucidate the metal propagation through solid electrolytes. Hence, the goal of this study is to establish a novel, in situ, non-invasive technique to characterize the mechanical and microstructural properties of LLZO before, during, and after the CCD has been exceeded. To this end, an acoustic technique was employed to enable the measurement of acoustic wave speed orthogonal to Li-ion current in Li-LLZO-Li symmetric cells. As Li dendrites propagate, the introduction of flaws or cracks filled with a relatively low elastic modulus phase $\left(E_{\mathrm{Li}}=\right.$ 13.0 GPa) compared to the LLZO parent phase $\left(E_{\text {LLZO }}=\sim 150 \mathrm{GPa}\right)$ results in a measurable decrease in acoustic wave speed. Thus, correlation with a reduction in wavespeed or elastic modulus upon Li dendrite propagation through LLZO will shed light on the phenomena that govern the CCD. We also believe this technique is applicable to other solid electrolytes and maybe useful in characterizing the mechanical integrity of ceramic electrolyte-ceramic electrode interfaces during cycling.

\section{Experimental procedure}

\subsection{Specimen preparation:}

Cubic $\mathrm{Li}_{6.25} \mathrm{Al}_{0.25} \mathrm{La}_{3} \mathrm{Zr}_{2} \mathrm{O}_{12}$ (LLZO) was calcined from starter powders of $\mathrm{Al}_{2} \mathrm{O}_{3}$ (99.9\%, AP-212, Mager Scientific, Dexter MI), $\mathrm{Li}_{2} \mathrm{CO}_{3}(99.0 \%, 36225$, Alfa Aesar, Ward Hill MA), $\mathrm{La}(\mathrm{OH})_{3}\left(99.95 \%\right.$, 43123, Alfa Aesar), and $\mathrm{ZrO}_{2}$ nanopowder $(99.9 \%$, 40N0801, Inframat Advanced Materials, Manchester CT). A 5 at\% excess lithium precursor 
was added to the stoichiometric quantities of precursors to allow for lithium loss during calcination. The doping concentration of alumina was chosen to maximize the Li-ion conductivity [9]. A precursor powder batch of $80 \mathrm{~g}$ was milled in a $500 \mathrm{~mL}$ agate lined jar (01.462.0225, Retsch $\mathrm{GmbH}$, Haan, Germany) with $50 \mathrm{~g}$ of $10 \mathrm{~mm}$ agate spherical media (Retsch $\mathrm{GmbH}$, Haan, Germany) at 350 RPM for $8 \mathrm{~h}$ in a planetary ball mill (PM100, Retsch $\mathrm{GmbH}$ ), with 5 minute intervals of milling followed by 5 min rest and reversal of rotation direction. The mixed powders were removed from the jar with a stainless steel spatula and passed through a $425 \mu \mathrm{m}$ sieve $(0488110 \mathrm{R}$, Fisher Scientific) to break up the larger agglomerates.

Prior to calcination, the mixed powders were pressed into $25.4 \mathrm{~mm}$ diameter pucks of $10 \mathrm{~g}$ each with $9000 \mathrm{~N}$ force for 2 minutes. Three $10 \mathrm{~g}$ pucks per batch were placed on a MgO boat and calcined in a tube furnace (STF 15/180, Carbolite LTD, Derbyshire, United Kingdom) with a $50 \mathrm{~mm}$ diameter alumina tube (EQ-TA-50D-M1000LD and EQ-FL-50, MTI Corporation, Richmond CA) and $2 \mathrm{~L} \mathrm{~min}^{-1}$ flowing dry air, heated to $1000^{\circ} \mathrm{C}$ at $100^{\circ} \mathrm{C} \mathrm{h}^{-1}$, held at $1000^{\circ} \mathrm{C}$ for $4 \mathrm{~h}$, and cooled to room temperature at $240^{\circ} \mathrm{C} \mathrm{h}^{-1}$. The resulting calcined pucks, each between $7.71 \mathrm{~g}$ and $7.76 \mathrm{~g}$, exhibited a core yolk of pale yellow material extending to the surface. The yellow material and the immediate surrounding material was manually removed, approximately $25 \%$ of the mass of each puck, and the remaining white cubic LLZO material of the pucks were ground and sieved through a $75 \mu \mathrm{m}$ sieve (0488110BB, Fisher Scientific). The ground and sieved cubic LLZO powder was milled for 30 minutes in an $80 \mathrm{~mL}$ agate line jar (01.462.0197, Retsch $\mathrm{GmbH}$ ) with 6 x $10 \mathrm{~mm}$ agate spherical media at $350 \mathrm{RPM}$, with 5 minute intervals of milling followed by 5 min rest and reversal of rotation direction. 
Each specimen was hot pressed from 8 - $10 \mathrm{~g}$ of milled LLZO powder from a single calcination batch in a graphite foil (99.8\%, 42953, Alfa Aesar) lined die. The powder was cold pressed at $62 \mathrm{MPa}$ or $16 \mathrm{MPa}$ for $30 \mathrm{~min}$, then sintered at $1100^{\circ} \mathrm{C}$ and the same $62 \mathrm{MPa}$ or $16 \mathrm{MPa}$ for $1 \mathrm{~h}$ by rapid induction hot press (IH15A, Across International, Livingston NJ). The sintered specimens were each sanded with 1500-grit paper to remove the graphite foil from the surface.

After removal of graphite foil, the sides of the specimen were secured with mounting wax (MWH135 South Bay Technology, San Clemente, CA) and the cylinder edges were cut by slow speed diamond saw (11-2180, Buehler, Lake Bluff, IL) at 100 RPM to create a rectangular cross section of approximately $8-9 \mathrm{~mm}$ per side. Each of the 6 faces of the parallelepiped specimen was polished with $15 \mu \mathrm{m}$ diamond paste and glycol-based diamond extender on a technotron polishing cloth (diamond paste 810915, extender 811-004, cloth 812-452, Leco Corp., St. Joseph, MI) using an automatic polishing wheel (LP 900A machine and 150 fixture, South Bay Technology).

\subsection{Materials characterization:}

A sample of the white calcined powder from each calcination batch and each of the densified specimens were examined by X-ray diffraction (Rotating Anode X-Ray Diffractometer, Rigaku, Tokyo, Japan) with a copper anode and graphite monochromater at $40 \mathrm{kV}$ and $100 \mathrm{~mA}$ to determine phase purity, as compared to cubic LLZO in the literature, $\mathrm{Li}_{6.5} \mathrm{La}_{3}\left(\mathrm{Ta}_{0.55} \mathrm{Zr}_{1.45}\right) \mathrm{O}_{12}($ ISCD 183686) [13].

Fractured or cut and polished surfaces of the LLZO specimens were imaged by SEM (Quanta 200, FEI, Hillsboro, Oregon) at $15 \mathrm{kV}$ and $0.31 \mathrm{nA}$. 


\subsection{Cycling experiment}

Cycling was performed within an argon glovebox with less than $0.25 \mathrm{ppm}$ oxygen content. The LLZO specimen consists of a rectangular parallelepiped shaped solid electrolyte with a height taller than that the face of an ultrasonic transducer and cross section of approximately $9 \mathrm{~mm} \times 9 \mathrm{~mm}$. Prior to cycling, each of the approximately 9 $\mathrm{mm} \times 9 \mathrm{~mm}$ faces that were to be in contact with lithium metal were sanded with a sheet of 1500-grit sandpaper inside the glovebox immediately before assembling. The lithium metal (10769, Alfa Aesar) was scraped with a stainless steel spatula to expose a fresh metallic surface, then a piece was cut to fit onto each sanded specimen surface. A piece of nickel-based foil (ASTM A753 Type 4) was placed on the outside of the lithium as an electrical connection. The entire stack (Ni-Li-LLZO-Li-Ni) was placed inside a Teflon sleeve with a cutout window, and compressed between two $12.7 \mathrm{~mm}$ nickel rods with manual force (Figure 1a). Proper contact between the LLZO and the Li metal, with reasonable bulk and contact resistance, was confirmed by electrochemical impedance spectroscopy (EIS) from $7 \mathrm{MHz}$ to $1 \mathrm{~Hz}$ (VMP-300 hardware and EC-Lab V10.40 software, Bio-Logic SAS, France).

The stack was heated to $170^{\circ} \mathrm{C}$ and preconditioned by cycling at $\pm 0.01 \mathrm{~mA} \mathrm{~cm}^{-2}$ for 30 minutes in each direction, repeating for a total of 10 full cycles to reduce the contact resistance [6]. After cooling to room temperature, a change in contact resistance was measured by EIS.

Cycling was performed at room temperature in steps of $0.05,0.1,0.2,0.3$ and $0.4 \mathrm{~mA} \mathrm{~cm}^{-2}$. Each cycle was performed for 30 minutes forward and 30 minutes in reverse polarization, followed by a 5 min or 10 min pause between cycles with no 
potential. Cycling was continued until a short circuit was observed, identified by a sudden drop in cell potential to near $0 \mathrm{~V}$.

During cycling, changes in the LLZO acoustic properties were monitored by measuring the acoustic wavespeed using a longitudinal transducer (pulser-receiver 5073PR-15-U, transducer V110-RM, couplant SWC, Olympus NDT Inc., Walham MA), (Figure $1 \mathrm{~b}$ and $1 \mathrm{c}$ ). The transducer was secured onto the side of the specimen in a pulse-echo configuration with a rubber band. Pulse time through the specimen was recorded every 2 or 5 minutes, with 3 successive pulse-echo measurements $(200 \mathrm{~Hz}$ pulse frequency) averaged for each time step.

\section{Results}

\subsection{Acoustic analysis overview and cell design}

In this study, a new in situ non-destructive technique was developed to characterize the elastic properties of a solid electrolyte during DC cycling (Figure 1a and 1b). The solid electrolyte specimen is positioned between two metallic lithium electrodes,(Figure 1c) with the transducer oriented perpendicular to the ionic current to assure that only the solid electrolyte is interrogated. The pulse-echo technique uses an ultrasonic pulse generated by the transducer to pass through the specimen, and times for the echo off of the opposite surface. The wavespeed may be used to not only determine the elastic properties of LLZO [14,15], but also observe microstructural changes $[16,17]$. With an accumulation of microcracks or other lower modulus defects, the wavespeed will decrease measurably, and the change in wavespeed may be used to reveal the microstructural changes associated with solid electrolyte degradation. 

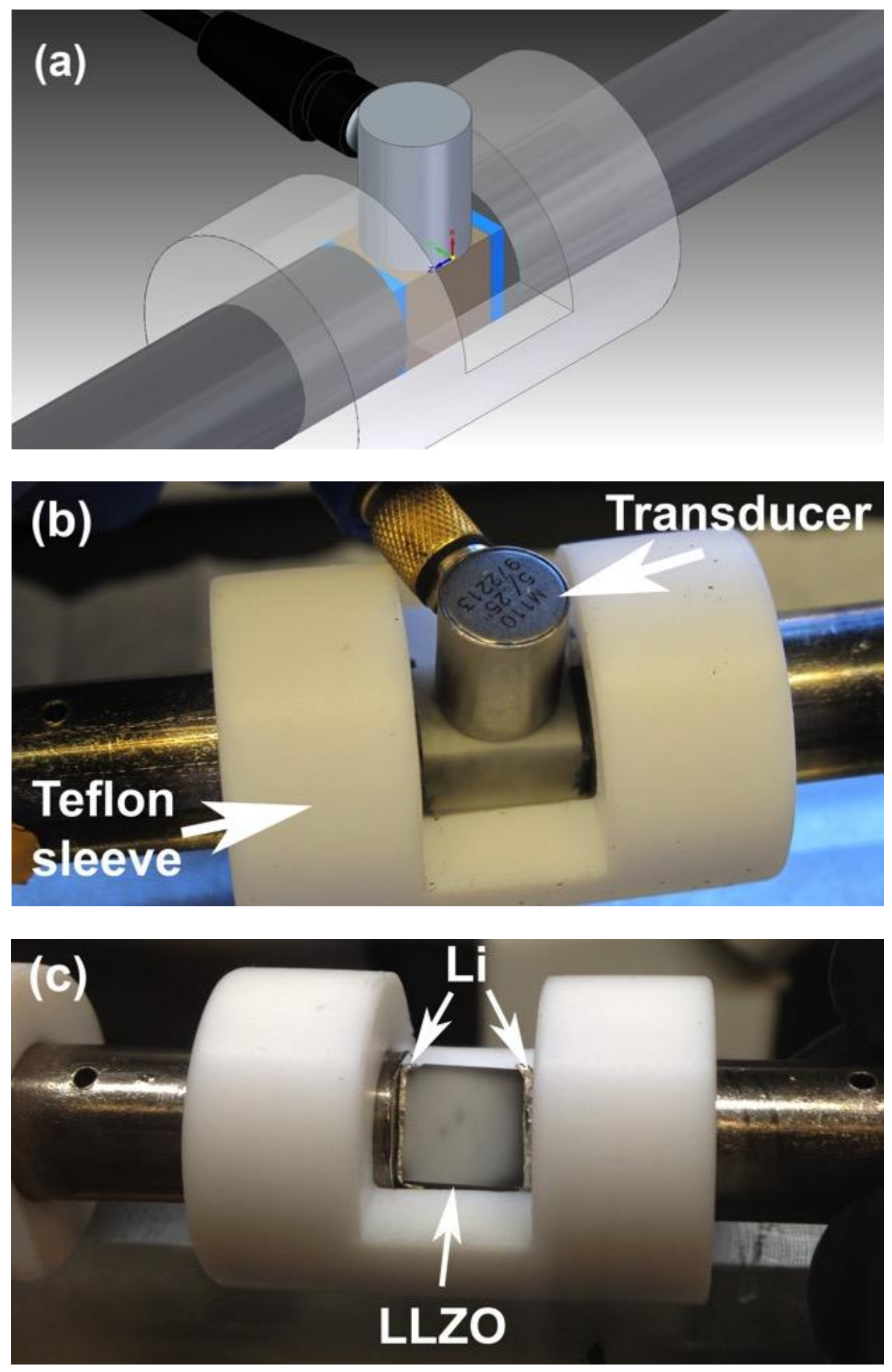

Figure 1. Cycling specimen in fixture (a) schematically represented, (b) with transducer on side of LLZO specimen, (c) and as assembled with lithium metal at each end. 


\subsection{XRD analysis before cycling}

The in-situ monitoring technique was validated with high purity cubic LLZO specimens. The as-prepared LLZO sintered specimens are consistent with cubic phase garnet (Figure 2), with a reference pattern based on previous literature of cubic $\mathrm{Li}_{6.5} \mathrm{La}_{3}\left(\mathrm{Ta}_{0.55} \mathrm{Zr}_{1.45}\right) \mathrm{O}_{12}$ [13]. A minor impurity phase of less than $1 \mathrm{wt} \%$ was observed by XRD in each sample, matched to the pattern of $\mathrm{La}_{2} \mathrm{Li}_{0.5} \mathrm{Al}_{0.5} \mathrm{O}_{4}[18]$, as indicated in Figure 2. The mass density of the specimens is greater than $4.95 \mathrm{~g} \mathrm{~cm}^{-3}$ (Table 1), with a corresponding relative density of 0.96 and 0.98 for the specimens, assuming theoretical density of $5.14 \mathrm{~g} \mathrm{~cm}^{-3}$ [14].

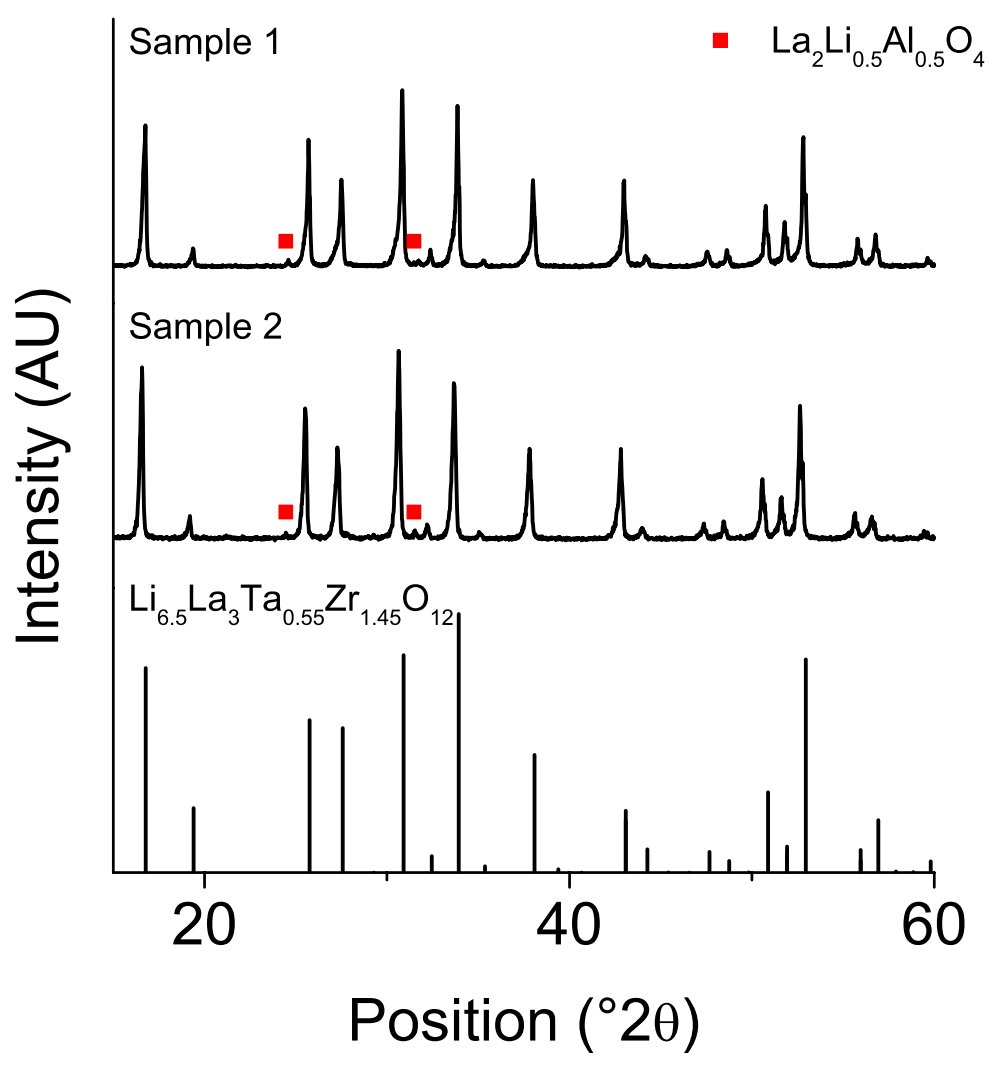

Figure 2. XRD of the LLZO specimens are consistent with primarily cubic phase LLZO, with less than $1 \mathrm{wt} \%$ of impurity phase, $\mathrm{La}_{2} \mathrm{Li}_{0.5} \mathrm{Al}_{0.5} \mathrm{O}_{4}$. 
densities of $0.05 \mathrm{~mA} \mathrm{~cm}^{-2}, 0.1 \mathrm{~mA} \mathrm{~cm}{ }^{-2}, 0.2 \mathrm{~mA} \mathrm{~cm}^{-2}, 0.3 \mathrm{~mA} \mathrm{~cm}^{-2}$, and $0.4 \mathrm{~mA} \mathrm{~cm}^{-2}$, or until short circuiting was observed. During electrochemical cycling of the half cells (LiLLZO-Li), short circuiting was observed as a sudden drop in voltage at what is defined as the critical current density (CCD) (Figure 4a and 4c). Prior to short circuiting, a deviation in ohmic behavior was observed, characterized by an increase in voltage, by as much as $50 \%$, at constant current. Both the sudden short circuit and the deviation from ohmic behavior have been observed before on LLZO specimens at similar current densities [6].

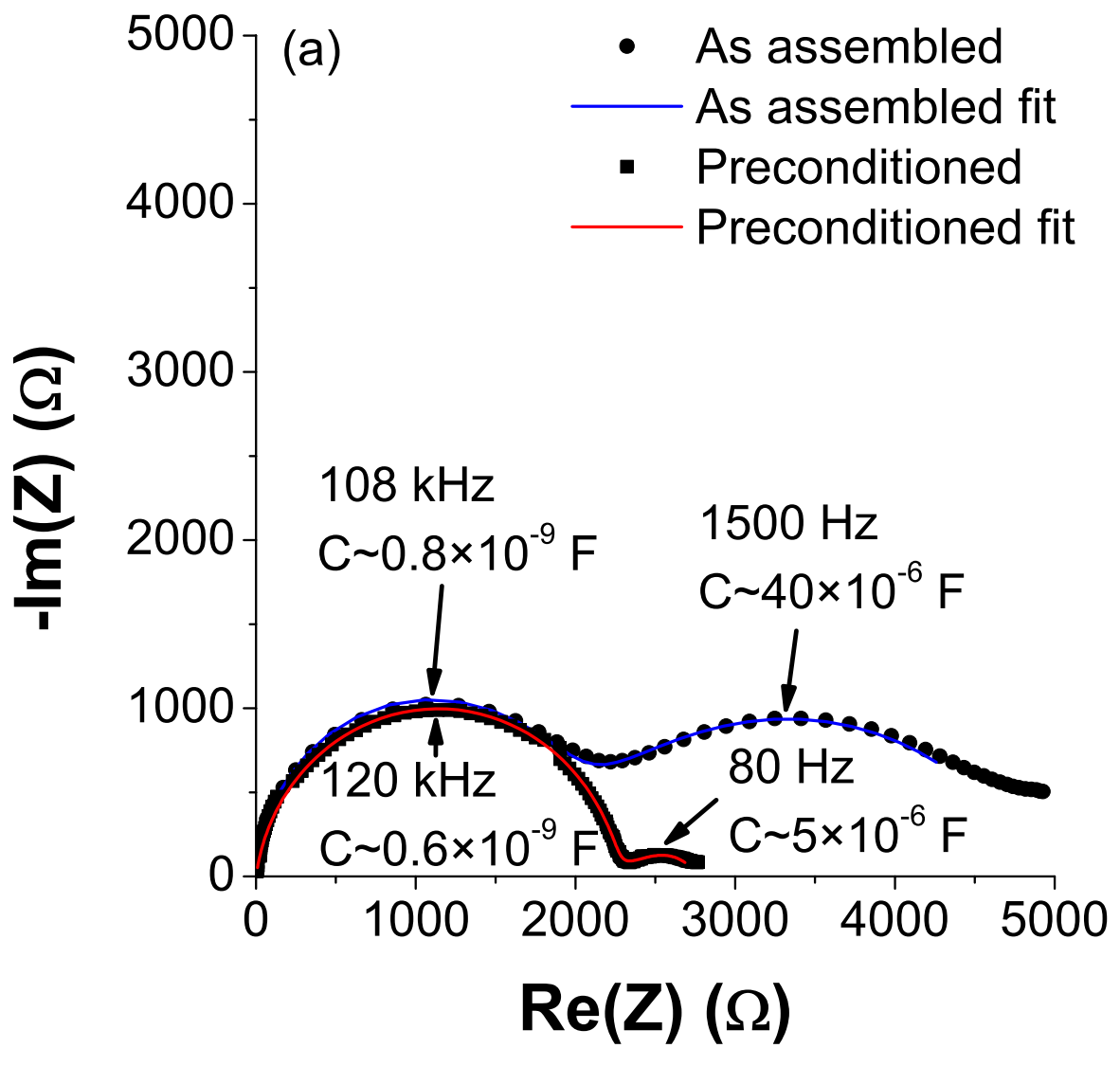




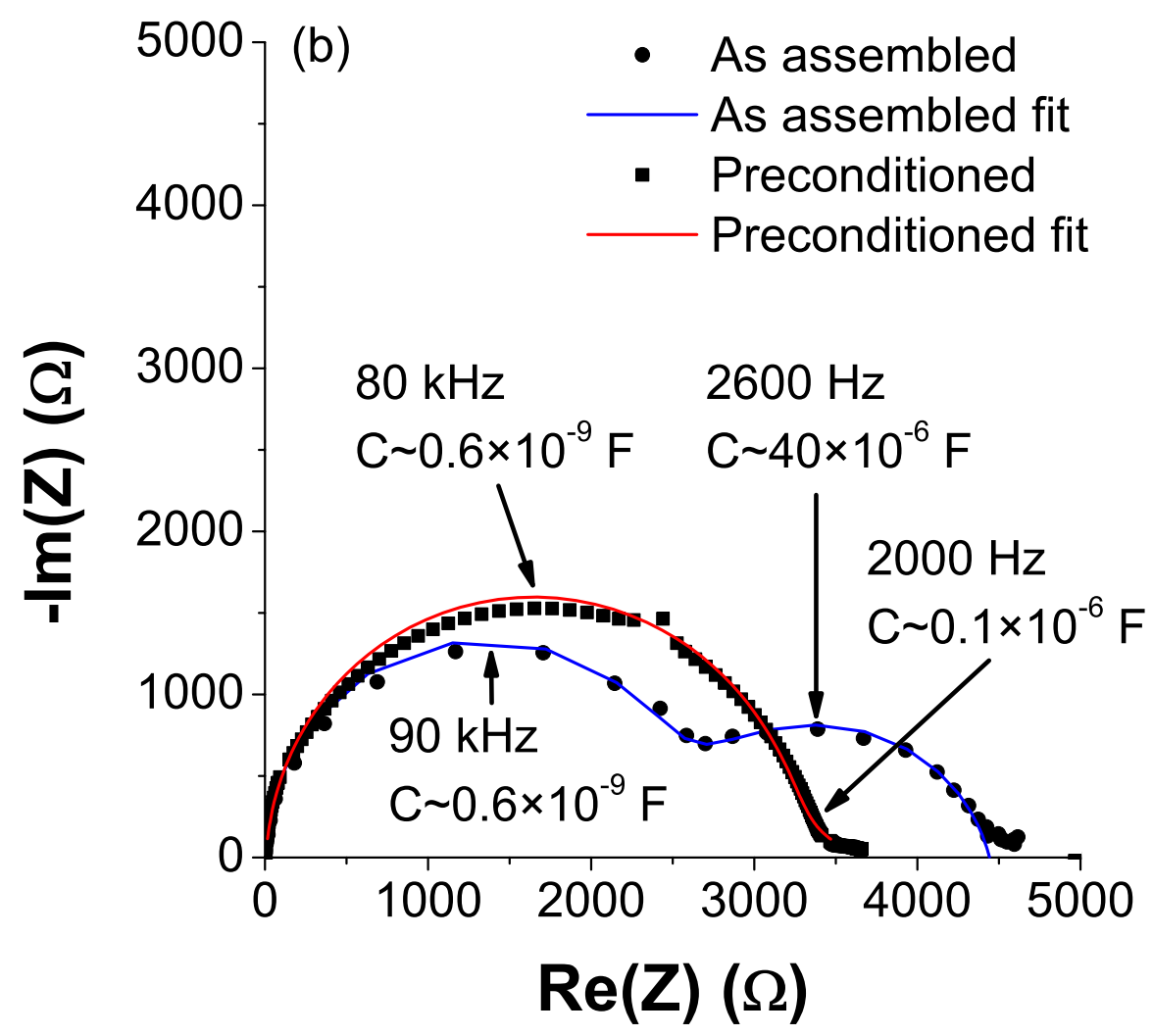

Figure 3. EIS of (a) sample 1 and (b) sample 2 indicate the preconditioning cycle increased bulk resistance a small to moderate amount (first semicircle), but decreased the contact resistance considerably (second semicircle).

\subsection{In situ Acoustic analysis}

Reported for the first time in this study, the wavespeed, $V_{l}$, decreased in the cycle prior to short circuiting of the LLZO specimen by $0.1-0.2 \%$ (Figure 4). In specimen 1 , a rapid decrease in $V_{l}$ during the $-0.2 \mathrm{~mA} \mathrm{~cm}^{-2}$ cycle in specimen 1 (Figure $4 \mathrm{~b}$ ) is indicative of a crack forming before the specimen is electrically short circuited, i.e. propagation of a Li dendrite. Similarly in specimen 2, a progressive decrease in $V_{\text {/ was }}$ measured during the $\pm 0.05 \mathrm{~mA} \mathrm{~cm}^{-2}$ cycles, followed by a rapid drop during the $+0.1 \mathrm{~mA}$ $\mathrm{cm}^{-2}$ cycle concurrent with the occurrence of an electrical short of the cell (Figure 4d). 


\subsection{Electrochemical measurements}

After preconditioning each specimen, the EIS scan (Figure 3) was fit to an equivalent circuit model of $\mathrm{Q} 1 / \mathrm{R} 1+\mathrm{Q} 2 / \mathrm{R} 2[1,6]$, an equivalent circuit previously fit to 1 $\mathrm{mm}$ thick LLZO specimens [6], where $Q$ is a constant phase element and $R$ is a resistor. Noise was noted in the EIS scan $(7 \mathrm{MHz}$ to $1 \mathrm{~Hz})$ at frequencies less than about $10 \mathrm{~Hz}$, which was excluded from the fitting. Resistor R1 and element Q1 represent the bulk and grain boundary, and resistor R2 and element Q2 represent the two Li-LLZO interfaces [6], listed in Table 1. The equivalent capacitances of the Q1 and Q2 elements from the model (EC-Lab) were $10^{-10} \mathrm{~F}$ to $10^{-9} \mathrm{~F}$ for the bulk (Q1), and $10^{-7} \mathrm{~F}$ to $10^{-5} \mathrm{~F}$ for the interface (Q2), as indicated in Figure 3. These specimen capacitance values are consistent with the expected range of capacitance for the ascribed phenomena of bulk resistance and sample-electrode interface $[6,19]$.

Conductivity was determined from the measured bulk resistance and the specimen dimensions. The measured conductivity of the specimens after preconditioning, $5.2 \times 10^{-4} \mathrm{~S} \mathrm{~cm}^{-1}$ and $4.8 \times 10^{-4} \mathrm{~S} \mathrm{~cm}^{-1}$ (Table 1), are consistent with previously reported LLZO conductivities of 4.0 to $5.7 \times 10^{-4} \mathrm{~S} \mathrm{~cm}^{-1}[2,20]$. The increase in bulk resistance after preconditioning in the lower relative density specimen 2 may be an indication of a reaction during the hold at $170^{\circ} \mathrm{C}$. For example, it is known that a relatively thin $\mathrm{Li}_{2} \mathrm{CO}_{3}$ contamination layer can form on the surface of LLZO that may affect ionic transport [21]. The contamination layer may form during polishing or other material trapped in the surface-penetrating porosity, discussed later in this section.

The conditioned specimens with measured conductivities (Table 1) were electrochemically cycled in forward and reverse polarization at increasing current 
However the initial indication of imminent failure was observable in the minutes prior to complete failure either during the same current density cycle or, for specimen 2 , during the previous current density cycle.
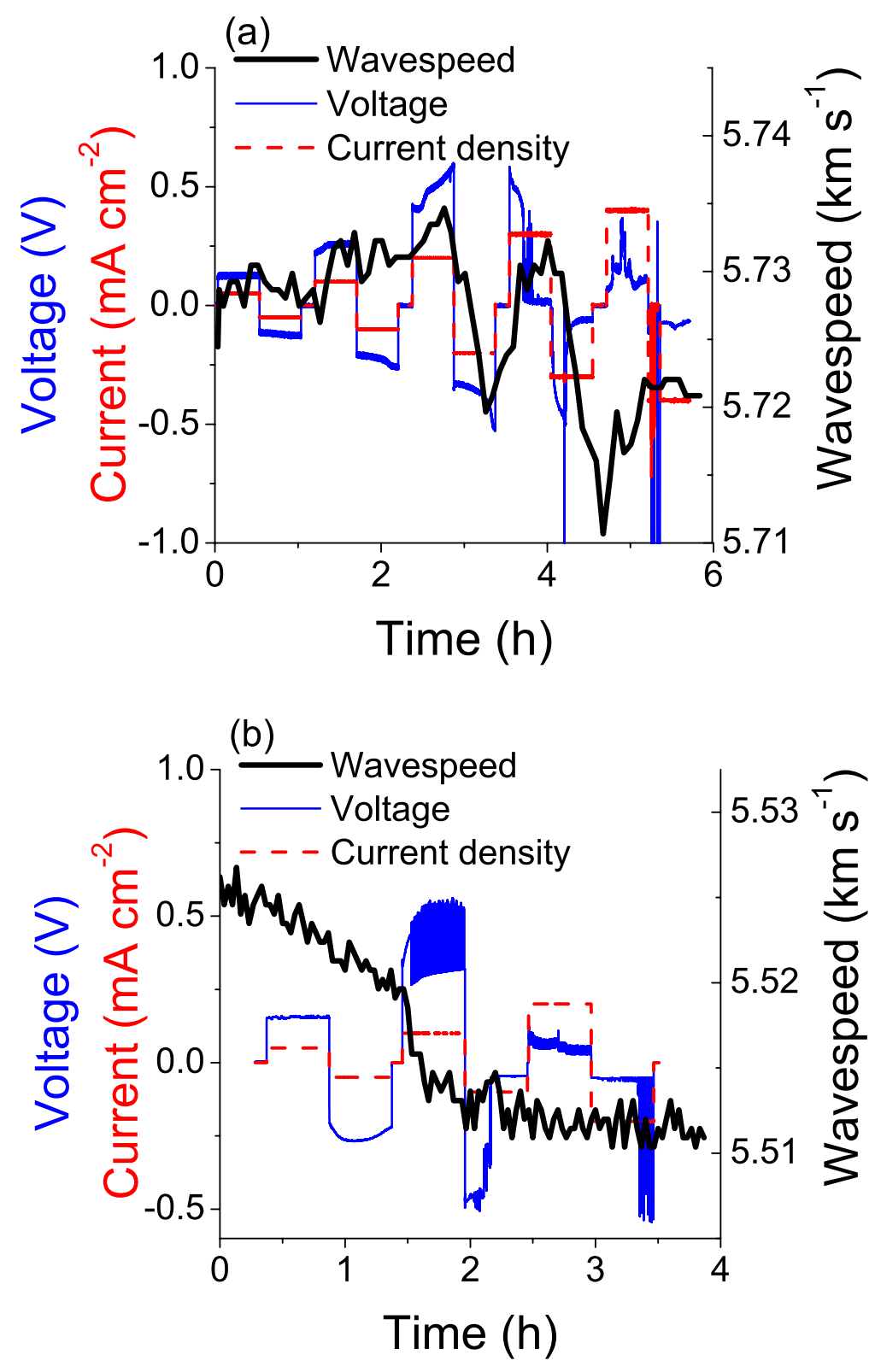

Figure 4. In specimen 1, the wavespeed decreased during the $0.2 \mathrm{~mA} / \mathrm{cm}^{2}$ cycle before short circuiting during the $0.3 \mathrm{~mA} / \mathrm{cm}^{2}$ cycle, indicated by the sudden drop in voltage. Similarly in specimen 2 (b), the wavespeed decreased during the 0.05 $\mathrm{mA} / \mathrm{cm} 2$ cycle before shorting during the $0.1 \mathrm{~mA} / \mathrm{cm}^{2}$ cycle. 


\subsection{SEM analysis}

After cycling, each specimen was cut and polished or fractured to observe the internal LLZO material. The observed porosity was consistent with the measured density of the specimens (Table 1), with differences in microstructure that may be responsible for the differences in critical current density between the specimens. In specimen 1 , the porosity was isolated and roughly spherical in shape (Figure $5 a-b)$. In specimen 2 , the porosity was partially connected, concentrated in roughly $20 \mu \mathrm{m}$ wide regions throughout the material, and tubular in shape (Figure 5c-d). This interconnected porosity may trap some contaminants from polishing, and may create a large flaw to initiate dendrite penetration at a lower current density. 

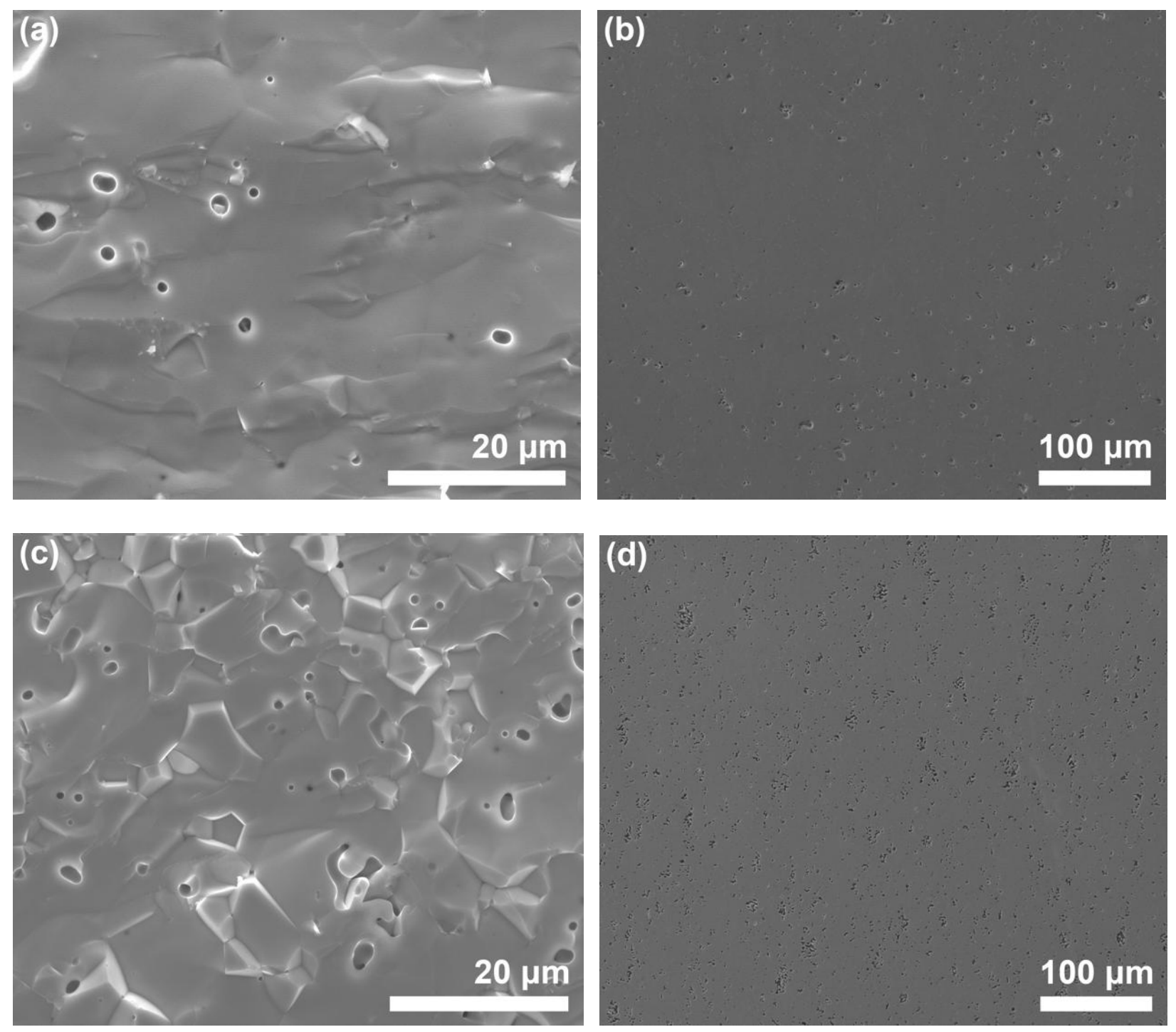

Figure 5. SEM fractured and polished surface micrographs of specimen $1(a, b)$ and specimen 2 (c, d). Porosity in specimen 1 is isolated and spherical, while the porosity in specimen 2 is tubular and partially interconnected. The interconnected porosity effectively increased the initial flaw size of specimen 2. 


\section{Discussion}

The implications of the observed changes in $V_{l}$ prior to short circuiting are significant with respect to the current density for crack and dendrite formation. The acoustic monitoring of the LLZO indicates the initial stages of failure occur during the minutes preceding the occurrence of short circuit, and failure of the electrolyte may be initiated at lower current density than reports based on the current at short circuiting. Without $V_{l}$ measurements or a similar technique, information about dendrite formation is unknown during electrochemical cycling. Based on observations of $V_{l}$ in this study, the rate of dendrite formation is dependent on the current density applied to the half cell, with a rapid progression towards failure when fracture has begun (Figure 4). The greatest part of the change in wavespeed for each specimen occurred during the $10-$ 25 minutes prior to short circuit, suggesting dendrite propagation is relatively fast at the measured critical current density. The critical current density for the more dense specimen 1 in this study, $0.3 \mathrm{~mA} \mathrm{~cm}^{-2}$, is similar to previously reported room temperature measurements for $1 \mathrm{~mm}$ thick specimens of LLZO fabricated by the same method [6]. The progression towards mechanical failure is particularly evident in specimen 2, in which the time rate of change in wavespeed was constant during the $0.05 \mathrm{~mA} \mathrm{~cm}^{-2}$ cycle (Figure 4d), consistent with dendrite and crack formation. The time rate of change in wavespeed abruptly increased at the start of the $0.1 \mathrm{~mA} \mathrm{~cm}^{-2}$ cycle, followed by a plateau after short circuit (Figure 4d), consistent with the dendrite and cracks quickly penetrating the specimen and a stopping of new dendrite formation/extension once the short circuit was formed through the specimen at a critical current density of $0.1 \mathrm{~mA} \mathrm{~cm}^{-2}$. Dendrite formation during the $0.05 \mathrm{~mA} \mathrm{~cm}^{-2}$ cycle also 
suggests the critical current density for specimen 2 may have been $0.05 \mathrm{~mA} \mathrm{~cm}^{-2}$ if the cycle time were longer than $1 \mathrm{~h}$, and that there is a time component to the critical current density.

The difference in critical current density between specimen 1 and 2 may be explained by a microstructural difference in the size and interconnection of porosity, with the interconnected porosity in specimen 2 acting as larger initial flaws from which the dendrites may grow. A previous investigation of sodium metal penetration in sodium beta alumina solid electrolyte observed a relationship between current density $i_{c r}$, fracture toughness $K_{C}$, viscosity of the metal $\eta$, a constant $A$, and the width and length of the dendrite filament $c$ and $/[22,23]$, where

$$
i_{c r}=\frac{A K_{C}^{4}}{\eta} \frac{c^{2}}{l^{3}}
$$

The relationship between the initial flaw size, captured in the $\frac{c^{2}}{l^{3}}$ term in equation 1 , and critical current density, is evident, and the partially interconnected tubular porosity in specimen 2 would be a relatively large value for the flaw size term. Therefore, the decreased critical current density in specimen 2 may be anticipated from the flaw size in the specimen and the fracture-controlled failure mode. The fracture-controlled failure also suggests the elimination of porosity and surface cracks as a method to increase the critical current density of LLZO, and is consistent with recent observations of improved critical current density with the elimination of porosity [24].

The changes in wavespeed observed are consistent with the introduction of microcracks in the specimen, and not just the creation of LLZO with lithium metal. For comparison of experimental results to model systems, a fractional change in the 
Young's modulus, $E$, is often reported in literature for specimens both with microcrack damage and for composites. In this study, Young's modulus may be calculated from $V_{l}$ if we assume the bulk material is isotropic, and use Poisson's ratio based on reported literature values [25]. The polycrystalline LLZO in this study was sintered from randomly oriented milled powder, and may be assumed as an isotropic solid. Poisson's ratio, $v$, is assumed for the uncycled LLZO from the average literature value, $v=0.2655$ [15]. From these assumptions, $E$ may be calculated from the measured $V_{l}$ as

$$
E=V_{l}^{2} \frac{\rho(1+v)(1-2 v)}{(1-v)}
$$

where $\rho$ is the mass density of the specimen and $v$ is Poisson's ratio. However, $v$ is also a function of microcracking, depending upon if the microcracks are open or have been compressed closed [26,27]. For open microcracks, the relative changes in $E$ and $v$ were approximated for small changes by Walsh [26], with good agreement with experiments [27], as

$$
\frac{v_{0}}{E_{0}}=\frac{v}{E}
$$

where the subscript 0 denotes the initial (uncycled) value. Furthermore, equation (3) has been shown to hold for non-random orientation cracks [28]. One may argue that the lithium dendrite effectively acts as a closing force on the microcrack, lessening or eliminating any change in $v$ [26]. Therefore, the actual $v$ of a cycled specimen should be effectively bounded by the initial $v_{0}$ of uncycled LLZO and by the result of equation (3).

Calculating the $E$ of specimen 1 from equation 2 as an example, the relative change in $E$ may be compared to literature for composite (Li-LLZO) or for microcrack damaged specimens. The average $V_{l}$ measured during the initial and final 30 minutes 


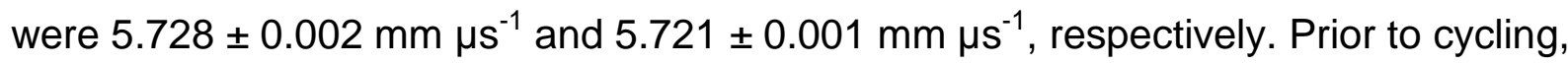
$E_{0}=133.19 \pm 0.09 \mathrm{GPa}$, where the subscript 0 denotes the initial (uncycled) value. The uncycled $E_{0}$ of specimen 1 is somewhat less than the $E_{0}$ of 146 - 154 GPa reported for fully dense Al-doped LLZO [14], but generally in agreement with a specimen consisting of 0.03 volume fraction porosity, $P$. Porosity reduces the $E$ of a brittle solid by the empirical relationship, $E=E_{\text {Dense }} \exp (-b P)$, where $b$ is typically between 2 and 6 [29]. A value of about 3.5 for $b$ would result in the observed difference. After cycling, $E$ of specimen 1 is reduced by microcracking. Applying the bounds of $v$ described in the previous paragraph, the $E$ for the cycled specimen 1 is between $132.87 \pm 0.03 \mathrm{GPa}$ (using equation (2) and constant $v$ ) and $133.03 \pm 0.03 \mathrm{GPa}$ (simultaneously using equations (2) and (3)), or a relative fraction reduction, $1-E / E_{0}$, of 0.0012 to 0.0024 .

The observed change in $E$ of LLZO is not consistent with a composite of LLZO and lithium metal. Assuming a composite, this measured modulus after cycling, $E_{c}$, would require an expected volume fraction of lithium metal, Vr. The Hashin particulate model, equation (4) [30-34], has previously been used to predict the $E_{c}$ of brittle (ceramic-like) composites with metal particle additions [34], and may also be used to determine expected volume fraction of lithium metal, $V r$, from the measured composite modulus,

$$
E_{c}=E_{m} \frac{\left(E_{m} V_{m}+E_{r}\left\{V_{r}+1\right\}\right)}{\left(E_{r} V_{m}+E_{m}\left\{V_{r}+1\right\}\right)}
$$

where $E_{m}$ is the matrix LLZO modulus and $V_{m}$ is the matrix volume fraction. For lithium metal, the $E$ is $12.6 \mathrm{GPa}$ at $\mathrm{OK}$, and approximately $10.5 \mathrm{GPa}$ when extrapolated to room temperature [35-37]. For a composite, the use of equation (3) with the assumption of 
microcracks would not apply, thus the composite modulus after cycling would be $E_{c}=$ 132.87 GPa. Applying $E_{m}=133.19 \mathrm{GPa}$ and $E_{c}=132.87 \mathrm{GPa}$ from equation $(2), E_{r}=$ 10.5 GPa from literature [35-37], and based on the volume of specimen 1 (table 1) and lithium density of $0.534 \mathrm{~g} \mathrm{~cm}^{-3}$, equation (4) predicts $0.58 \mathrm{mg}$ of lithium metal in specimen 1 to create a composite of the measured stiffness. To move $0.58 \mathrm{mg}$ of lithium would require $8.1 \mathrm{C}$ of charge. However, the specimen damage was complete after the $0.3 \mathrm{~mA} \mathrm{~cm}^{-2}$ cycle, after which only $1.8 \mathrm{C}$ of charge had been passed through the specimen (Figure 4). Even if all the charge had created lithium particles inside the LLZO, the amount of lithium inserted would not be sufficient to reduce the $E$ of the composite to the measured value. A composite LLZO-Li material cannot account for the observed reduction in $E$ of LLZO.

The relative reduction in $E$ of $L L Z O$ is consistent with the anticipated reduction based on published relationships on the moduli of cracked solids. Previous investigations have expressed the reduction in $E$ of a cracked body as

$$
E=E_{0}(1-f \varepsilon)
$$

where $f$ is a function of the spatial orientation of the cracks and $\varepsilon$ is a crack damage parameter $[28,38]$. The crack damage parameter $\varepsilon$ is also referred to as the crack density [39]. Equation (5a) implies that, for a given spatial orientation factor of microcracks $f$, the $E$ of a material decreases linear with increasing density of microcracks $\varepsilon$. Rearranged, equation (5a) shows the relative reduction in $E$ as

$$
1-\frac{E}{E_{0}}=f \varepsilon
$$


Therefore if cracks in the microstructure of LLZO are responsible for the change in $E$, the product of $f$ and $\varepsilon$ should correspond to the observed relative reduction in $E$.

The exact orientation of the full set of cracks in the microstructure is unknown, however the general location is known. Previous work by Ren et al. showed a set of cracks in different orientations where the dendrite penetrated the LLZO, but grouped around the location of the dendrite [7]. For the purposes of this study, the spatial orientation is assumed to be random. This assumption is an oversimplification, but should allow a calculation to determine if the observed change in wavespeed is of the correct order of magnitude to be accounted for by cracks. A similar texturing of microcracks was observed in $\mathrm{Gd}_{2} \mathrm{O}_{3}$ and $\mathrm{HfO}_{2}$, however the measured changes fit the predicted values well [27]. The spatial orientation function $f$ for a random penny-shaped crack and the parameter $\varepsilon$ are defined as

$$
\begin{aligned}
& f=\frac{16}{45} \frac{\left(1-v_{0}^{2}\right)\left(10-3 v_{0}\right)}{\left(2-v_{0}\right)} \\
& \varepsilon=\frac{2}{\pi}\left(\frac{\left\langle A^{2}\right\rangle}{\langle P\rangle}\right) N_{V}
\end{aligned}
$$

where $\left\langle A^{2}\right\rangle$ is the average area of the crack, $\langle P>$ is the average crack perimeter, and $N_{V}$ is the volume number density of cracks [38-42]. The function $f$ here was calculated by Budiansky and O'Connell for random elliptical cracks [42]. Roughly similar values of $E / E_{0}$ were calculated for other $f$ functions associated with different orientations and shapes by Laws and Brockenbrough [40], and by numerical simulation by Shen and Li [39]. Thus the use of the $f$ function for random orientation of elliptical cracks, while not 
exact, is a reasonable assumption for a rough approximation, as the choice of $f$ function is not likely to result in a large change.

Based on previous studies of cycled LLZO, the dendrites appear in the bulk material as dark linear spots, and to a rough approximation are of the same diameter as the grain size $[6,7]$. Using these previous observations of dendrites in LLZO [6,7], some assumptions may be made to estimate the size and number density of cracks in a specimen of LLZO, and the resulting change in E. Based on the observation of dendrites in a collection of microcracks of similar length as the grain size [7] and presenting in the bulk as a linear spot roughly $1 \mathrm{~mm}$ wide [6], the dark linear spot may be examined as a collection of dendrite-filled cracks within an area $1 \mathrm{~mm}$ wide, one grain thick, and extended through the length of the specimen, $9.44 \mathrm{~mm}$. Each crack may be assumed as penny-shaped and of the same diameter as the grains. We assume half of the grains in the area of the dendrite are cracked, or a linear filling fraction of 0.5 . At an approximate grain size of $2.5 \mu \mathrm{m}$ and a linear filling fraction of 0.5 , the dark spot introduced a collection of $N_{V}=980000$ cracks $\mathrm{cm}^{-3}$ to the specimen, with each crack of area $\left\langle A^{2}\right\rangle=4.9 \mu \mathrm{m}^{2}$ and perimeter $\langle P\rangle=7.9 \mu \mathrm{m}$. Applying these approximate values to equations (6) and (7), and then solving equation (5b) for the relative change in $E, 1-$ $E / E_{0}=f \varepsilon=0.0026$. This approximate solution to equation (5b) is consistent with the measured $1-E / E_{0}$ of 0.0012 to 0.0024 , indicating the observed change in modulus of LLZO is consistent with a cracked solid.

The difference of about $8 \%$ between the calculated and measured $E / E_{0}$ is remarkably small, given the assumptions above for the spatial orientation function $f$, the exact size of the cracks, and the filling fraction. With the set of assumptions applied, 
particularly the filling fraction, an error of $50 \%$ is assumed on the calculation. In this work, the calculation is only to illustrate that the observations are consistent with the general expectation for a cracked solid, and not an exact solution.

Future use of the acoustic monitoring technique may be applied to other solid electrolytes or examine the microstructure of a specimen after an observed reduction in $V_{l}$ but before short circuit occurs. Future development and examination of LLZO may concentrate on the complete elimination of porosity and production of transparent LLZO with larger grain sizes as a method to suppress dendrite penetration, with dendrite monitoring possible by acoustic pulse-echo.

\section{Conclusions}

The acoustic pulse-echo technique has been demonstrated as an in-situ method to monitor the degradation of solid state electrolyte materials immediately prior to short circuit. A decrease in the stiffness of the LLZO was observed in the minutes prior to failure, with the rate of stiffness decrease dependent on the applied current density. The observed decrease in stiffness of LLZO is consistent with established theory for a cracked solid with dendrites of the approximate dimensions observed after failure. This technique may provide a method to detect imminent failure and allow engineered controls prior to complete short circuit in a battery cell, such as a tool for assembled batteries to find and isolate failed or failing cells. The monitoring of change in stiffness may be used as a tool to stop cycling and examine the intermediate stage of failure in a cell prior to short circuit. Crack initiation and extension occurs prior to complete failure, and methods to observe the failure as it occurs will be necessary for further development of solid state electrolytes. 


\section{Acknowledgement}

This work was supported by the U.S. Department of Energy (DOE) Office of

Energy Efficiency and Renewable Energy (EERE) Vehicle Technologies Office (VTO)

and Advanced Battery Material Research (BMR) programs Grant No. DE-EE0991-1663.

\section{References}

[1] R. Murugan, V. Thangadurai, W. Weppner, Fast Lithium Ion Conduction in Garnet-Type Li7La3Zr2O12, Angew. Chemie Int. Ed. 46 (2007) 7778-7781. doi:10.1002/anie.200701144.

[2] E. Rangasamy, J. Wolfenstine, J. Sakamoto, The role of Al and Li concentration on the formation of cubic garnet solid electrolyte of nominal composition Li7La3Zr2O12, Solid State Ionics. 206 (2012) 28-32. doi:10.1016/j.ssi.2011.10.022.

[3] J. Sakamoto, Super-ionic Conducting Oxide Electrolytes, in: N.J. Dudney, W.C. West, J. Nanda (Eds.), Handb. Solid State Batter., Second Edi, World Scientific, Hackensack, NJ, 2016: pp. 391-414. doi:10.1142/9789814651905_0012.

[4] W.H. Meyer, Polymer Electrolytes for Lithium-Ion Batteries, Adv. Mater. 10 (1998) 439-448. doi:10.1002/(SICI)1521-4095(199804)10:6<439::AIDADMA439>3.0.CO;2-I.

[5] S. Ohta, T. Kobayashi, T. Asaoka, High lithium ionic conductivity in the garnettype oxide Li7-X La3(Zr2-X, NbX)O12 (X=0-2), J. Power Sources. 196 (2011) 3342-3345. doi:10.1016/j.jpowsour.2010.11.089.

[6] A. Sharafi, H.M. Meyer, J. Nanda, J. Wolfenstine, J. Sakamoto, Characterizing the $\mathrm{Li}-\mathrm{Li}$ La3Zr2O12 interface stability and kinetics as a function of temperature and current density, J. Power Sources. 302 (2016) 135-139.

doi:10.1016/j.jpowsour.2015.10.053.

[7] Y. Ren, Y. Shen, Y. Lin, C.-W. Nan, Direct observation of lithium dendrites inside garnet-type lithium-ion solid electrolyte, Electrochem. Commun. 57 (2015) 27-30. doi:10.1016/j.elecom.2015.05.001.

[8] K. Ishiguro, Y. Nakata, M. Matsui, I. Uechi, Y. Takeda, O. Yamamoto, et al., Stability of Nb-Doped Cubic Li7La3Zr2O12 with Lithium Metal, J. Electrochem. Soc. 160 (2013) A1690-A1693. doi:10.1149/2.036310jes.

[9] T. Thompson, A. Sharafi, M.D. Johannes, A. Huq, J.L. Allen, J. Wolfenstine, et al., A Tale of Two Sites: On Defining the Carrier Concentration in Garnet-Based lonic Conductors for Advanced Li Batteries, Adv. Energy Mater. 5 (2015) 1500096. doi:10.1002/aenm.201500096.

[10] M.P.J. Brennan, The failure of beta-alumina electrolyte by a dendritic penetration 
mechanism, Electrochim. Acta. 25 (1980) 621-627. doi:10.1016/00134686(80)87067-8.

[11] A. V. Virkar, L. Viswanathan, D.R. Biswas, On the deterioration of $B$ "-alumina ceramics under electrolytic conditions, J. Mater. Sci. 15 (1980) 302-308. doi:10.1007/BF02396777.

[12] L.C. Jonghe, L. Feldman, A. Beuchele, Slow degradation and electron conduction in sodium/beta-aluminas, J. Mater. Sci. 16 (1981) 780-786. doi:10.1007/BF00552217.

[13] A. Logéat, T. Köhler, U. Eisele, B. Stiaszny, A. Harzer, M. Tovar, et al., From order to disorder: The structure of lithium-conducting garnets Li7-xLa3TaxZr2-xO12 (x=0-2), Solid State lonics. 206 (2012) 33-38. doi:10.1016/j.ssi.2011.10.023.

[14] S. Yu, R.D. Schmidt, R. Garcia-Mendez, E. Herbert, N.J. Dudney, J.B. Wolfenstine, et al., Elastic Properties of the Solid Electrolyte Li 7 La 3 Zr 2012 (LLZO), Chem. Mater. 27 (2015) acs.chemmater.5b03854. doi:10.1021/acs.chemmater.5b03854.

[15] J.E. Ni, E.D. Case, J.S. Sakamoto, E. Rangasamy, J.B. Wolfenstine, Room temperature elastic moduli and Vickers hardness of hot-pressed LLZO cubic garnet, J. Mater. Sci. 47 (2012) 7978-7985. doi:10.1007/s10853-012-6687-5.

[16] J.E. Ni, E.D. Case, R.D. Schmidt, C.-I. Wu, T.P. Hogan, R.M. Trejo, et al., Fracture mode, microstructure and temperature-dependent elastic moduli for thermoelectric composites of $\mathrm{PbTe}-\mathrm{PbS}$ with $\mathrm{SiC}$ nanoparticle additions, Philos. Mag. 93 (2013) 4412-4439. doi:10.1080/14786435.2013.833354.

[17] J.E. Ni, E.D. Case, Thermal Fatigue of Cast and Hot-Pressed Lead-AntimonySilver-Tellurium (LAST) Thermoelectric Materials, J. Electron. Mater. 42 (2013) 1382-1388. doi:10.1007/s11664-012-2254-8.

[18] F. Abbattista, M. Vallino, D. Mazza, Preparation and characterization of La2Li0.5AI0.5O4 with K2NiF4 structure, Inorganica Chim. Acta. 140 (1987) 147149. doi:10.1016/S0020-1693(00)81072-5.

[19] J.T.S. Irvine, D.C. Sinclair, A.R. West, Electroceramics: Characterization by Impedance Spectroscopy, Adv. Mater. 2 (1990) 132-138. doi:10.1002/adma.19900020304.

[20] Y. Zhang, F. Chen, R. Tu, Q. Shen, L. Zhang, Field assisted sintering of dense Alsubstituted cubic phase Li7La3Zr2O12 solid electrolytes, J. Power Sources. 268 (2014) 960-964. doi:10.1016/j.jpowsour.2014.03.148.

[21] H. Buschmann, J. Dölle, S. Berendts, A. Kuhn, P. Bottke, M. Wilkening, et al., Structure and dynamics of the fast lithium ion conductor "Li7La3Zr2O12," Phys. Chem. Chem. Phys. 13 (2011) 19378-19392. doi:10.1039/c1cp22108f.

[22] A. V. Virkar, The role of superimposed stresses on the degradation of solid electrolytes, J. Mater. Sci. 21 (1986) 859-865. doi:10.1007/BF01117365. 
[23] A. V. Virkar, L. Viswanathan, A three-dimensional approach to the electrolytic degradation of solid electrolytes, J. Mater. Sci. 18 (1983) 1202-1212. doi:10.1007/BF00551990.

[24] Y. Suzuki, K. Kami, K. Watanabe, A. Watanabe, N. Saito, T. Ohnishi, et al., Transparent cubic garnet-type solid electrolyte of Al2O3-doped Li7La3Zr2O12, Solid State Ionics. 278 (2015) 172-176. doi:10.1016/j.ssi.2015.06.009.

[25] J.F. Nye, Physical Properties of Crystals: Their representation by Tensors and matrices, Oxford University Press, New York, 1985.

[26] J.B. Walsh, The effect of cracks in rocks on Poisson's ratio, J. Geophys. Res. 70 (1965) 5249-5257. doi:10.1029/JZ070i020p05249.

[27] E.D. Case, The effect of microcracking upon the Poisson's ratio for brittle materials, J. Mater. Sci. 19 (1984) 3702-3712. doi:10.1007/BF02396943.

[28] X. Fan, E.D. Case, M.J. Baumann, The effect of indentation-induced microcracks on the elastic modulus of hydroxyapatite, J. Mater. Sci. 47 (2012) 6333-6345. doi:10.1007/s10853-012-6556-2.

[29] R.W. Rice, Porosity of Ceramics, Marcel Dekker, New York, 1998.

[30] R.D. Schmidt, E.D. Case, J.E. Ni, R.M. Trejo, E. Lara-Curzio, R.J. Korkosz, et al., High-temperature elastic moduli of thermoelectric SnTe1 $\pm x-y$ SiC nanoparticulate composites, J. Mater. Sci. 48 (2013) 8244-8258. doi:10.1007/s10853-013-7637-6.

[31] Z. Hashin, The Elastic Moduli of Heterogeneous Materials, J. Appl. Mech. 29 (1962) 143-150. doi:10.1115/1.3636446.

[32] E. Bedolla, J. Lemus-Ruiz, A. Contreras, Synthesis and characterization of MgAZ91/AIN composites, Mater. Des. 38 (2012) 91-98. doi:10.1016/j.matdes.2012.02.001.

[33] R. Couturier, D. Ducret, P. Merle, J.P. Disson, P. Joubert, Elaboration and characterization of a metal matrix composite: Al/AIN, J. Eur. Ceram. Soc. 17 (1997) 1861-1866. doi:doi:10.1016/S0955-2219(97)87873-9.

[34] R.D. Schmidt, E.D. Case, Z. Lobo, T.R. Thompson, J.S. Sakamoto, X.-Y. Zhou, et al., Influence of silver nanoparticle addition, porosity, and processing technique on the mechanical properties of Ba0.3Co4Sb12 skutterudites, J. Mater. Sci. 49 (2014) 7192-7212. doi:10.1007/s10853-014-8427-5.

[35] H.C. Nash, C.S. Smith, Single-crystal elastic constants of lithium, J. Phys. Chem. Solids. 9 (1959) 113-118. doi:0.1016/0022-3697(59)90201-X.

[36] T. Slotwinski, J. Trivisonno, Temperature dependence of the elastic constants of single crystal lithium, J. Phys. Chem. Solids. 30 (1969) 1276-1278. doi:10.1016/0022-3697(69)90386-2.

[37] J.P. Day, A.L. Ruoff, The variation of the elastic constants of lithium with temperature and pressure, Phys. Status Solidi. 205 (1974) 205-213. 
doi:10.1002/pssa.2210250118.

[38] Y. Kim, E.D. Case, The effect of surface-limited microcracks on the effective Young 's modulus of ceramics Part II Application of analysis to particular microcrack geometries, J. Mater. Sci. 28 (1993) 1901-1909. doi:10.1007/BF00595763.

[39] L. Shen, J. Li, A numerical simulation for effective elastic moduli of plates with various distributions and sizes of cracks, Int. J. Solids Struct. 41 (2004) 74717492. doi:10.1016/j.ijsolstr.2004.02.016.

[40] N. Laws, J.R. Brockenbrough, The effect of micro-crack systems on the loss of stiffness of brittle solids, Int. J. Solids Struct. 23 (1987) 1247-1268. doi:10.1016/0020-7683(87)90104-1.

[41] A. Hoenig, Elastic Moduli of a Non - Randomly Cracked Body, Int. J. Solids Struct. 15 (1979) 137-154.

[42] B. Budiansky, R.J. O'connell, Elastic moduli of a cracked solid, Int. J. Solids Struct. 12 (1976) 81-97. doi:10.1016/0020-7683(76)90044-5. 


\section{Figure Captions}

Figure 1. Cycling specimen in fixture (a) schematically represented, (b) with transducer on side of LLZO specimen, (c) and as assembled with lithium metal at each end.

Figure 2. XRD of the LLZO specimens are consistent with primarily cubic phase LLZO, with less than $1 \mathrm{wt} \%$ of impurity phase, $\mathrm{La}_{2} \mathrm{Li}_{0.5} \mathrm{Al}_{0.5} \mathrm{O}_{4}$.

Figure 3. EIS of (a) sample 1 and (b) sample 2 indicate the preconditioning cycle increased bulk resistance a small to moderate amount (first semicircle), but decreased the contact resistance considerably (second semicircle).

Figure 4. In specimen 1, the wavespeed decreased during the $0.2 \mathrm{~mA} / \mathrm{cm} 2$ cycle before short circuiting during the $0.3 \mathrm{~mA} / \mathrm{cm} 2$ cycle, indicated by the sudden drop in voltage. Similarly in specimen 2 (b), the wavespeed decreased during the $0.05 \mathrm{~mA} / \mathrm{cm} 2$ cycle before shorting during the $0.1 \mathrm{~mA} / \mathrm{cm} 2$ cycle.

Figure 5. SEM fractured and polished surface micrographs of specimen $1(a, b)$ and specimen 2 (c, d). Porosity in specimen 1 is isolated and spherical, while the porosity in specimen 2 is tubular and partially interconnected. The interconnected porosity effectively increased the initial flaw size of specimen 2. 
Table 1. LLZO Specimen conditions prior to cycling

\begin{tabular}{|l|r|r|}
\hline Specimen & 1 & 2 \\
& & \\
\hline Dimensions $(\mathrm{mm})$ & $9.442 \times 9.095 \times 8.987$ & $11.835 \times 9.287 \times 8.494$ \\
\hline Density $\left(\mathrm{g} \mathrm{cm}^{-3}\right)$ & 5.023 & 4.957 \\
\hline Bulk $\sigma\left(\mathrm{mS} \mathrm{cm}^{-1}\right)$ & 0.52 & 0.48 \\
\hline Bulk Q $\left(\mathrm{nF} \mathrm{s}^{\alpha-1}\right)$ & 1.2 & 0.67 \\
\hline Equiv. Bulk C $(\mathrm{nF})$ & 0.69 & 0.61 \\
\hline Bulk $\alpha$ & 0.95 & 0.99 \\
\hline Contact resistance $\left(\Omega \mathrm{cm}^{-2}\right)$ & 450 & 370 \\
\hline Contact Q $\left(\mu \mathrm{F} \mathrm{s}{ }^{\alpha-1}\right)$ & 71 & 14 \\
\hline Equiv. Contact C $(\mu \mathrm{F})$ & 4.5 & 0.11 \\
\hline Contact $\alpha$ & 0.54 & 0.51 \\
\hline
\end{tabular}

